\title{
Towards the development of rapid biofilm antibiotic sensitivity testing (BAST)
}

\author{
AL Quthami Khalid ${ }^{1 \star}$, Bassam O. AlJohny ${ }^{2}$ and Milton Wainwright ${ }^{3}$ \\ ${ }^{1}$ Regional Laboratory,MOH, Makkak, Saudi Arabia. \\ ${ }^{2}$ Biological Science Department, Faculty of Science, King Abdulaziz University, P, O, Box 80203, Jeddah 21589, \\ Kingdom of Saudi Arabia. \\ ${ }^{3}$ Department of Molecular Biology and Biotechnology, University of Sheffield, Sheffield, S10 2TN, United Kingdom.
}

Accepted 9 September, 2013

This study presents a modification of the antibiotic susceptibility testing (AST), which is a rapid means of determining the response of planktonic bacteria to different antimicrobial agents, for application to biofilms. Colony biofilm was first developed on a cellulose filter/membrane disc, over which an antibiotic disc was imposed. Zone of inhibition was measured after incubation on nutrient agar. Biofilms were not as susceptible to the test antibiotics as compared to the planktonic cultures. The results point to the possibility of this method as a rapid means for antibiotics for treating biofilm infections. Limitations and potential application for biofilm AST are discussed.

Key words: Biofilm, antibiotic susceptibility testing.

\section{INTRODUCTION}

The antibiotic susceptibility testing (AST) is a rapid means of determining the response of bacteria to different antimicrobial agents. AST utilizes the disc diffusion method, which is simple and widely used for sensitivity testing (Bauer et al., 1966; Clinical and Laboratory Standards Institute, 2009). Disc diffusion is very flexible, with a wide variety of antibiotic discs of varying concentrations available. This test effectively identifies organisms that are susceptible/resistant to certain antibiotics through visual means. Identification of effective antibiotics will lead to the successful treatment of bacterial infections. However, the test is conducted on planktonic cultures, which differ from biofilms in their response to antimicrobials in vivo (Jorgensen and Ferraro, 2009).

Studies show that $80 \%$ of infectious diseases are due to biofilm bacteria that are more difficult to treat than planktonic bacteria. (Lewis, 2005; Walters et al., 2003). Most biofilm antibiotic susceptibility tests utilized polystyrene wells for growing biofilms in vitro (Cerca et al., 2005; Ceri et al., 1999; Olson et al., 2002; Amorena et al., 1999). Results are not easily visualized compared to the disc assay. Therefore, it is necessary to develop a rapid test that will identify antibiotics for effectively treating biofilm infections.

This study presents a modification of the antibiotic susceptibility testing performed on planktonic cultures for application to biofilm cultures.

\section{MATERIALS AND METHODS}

Preparation of bacterial culture

Overnight cultures of Pseudomonas aeruginosa PA01 and Staphylococcus aureus $\mathrm{SH} 1000$ were prepared by dispersing a single colony in 5-ml test tubes in Mueller-Hinton broth (MHB). After incubation at $37^{\circ} \mathrm{C}$ in air with shaking, $500 \mu \mathrm{l}$ of the overnight cultures were transferred to a $50 \mathrm{ml}$ flask with $10 \mathrm{ml} \mathrm{MHB}$, and 


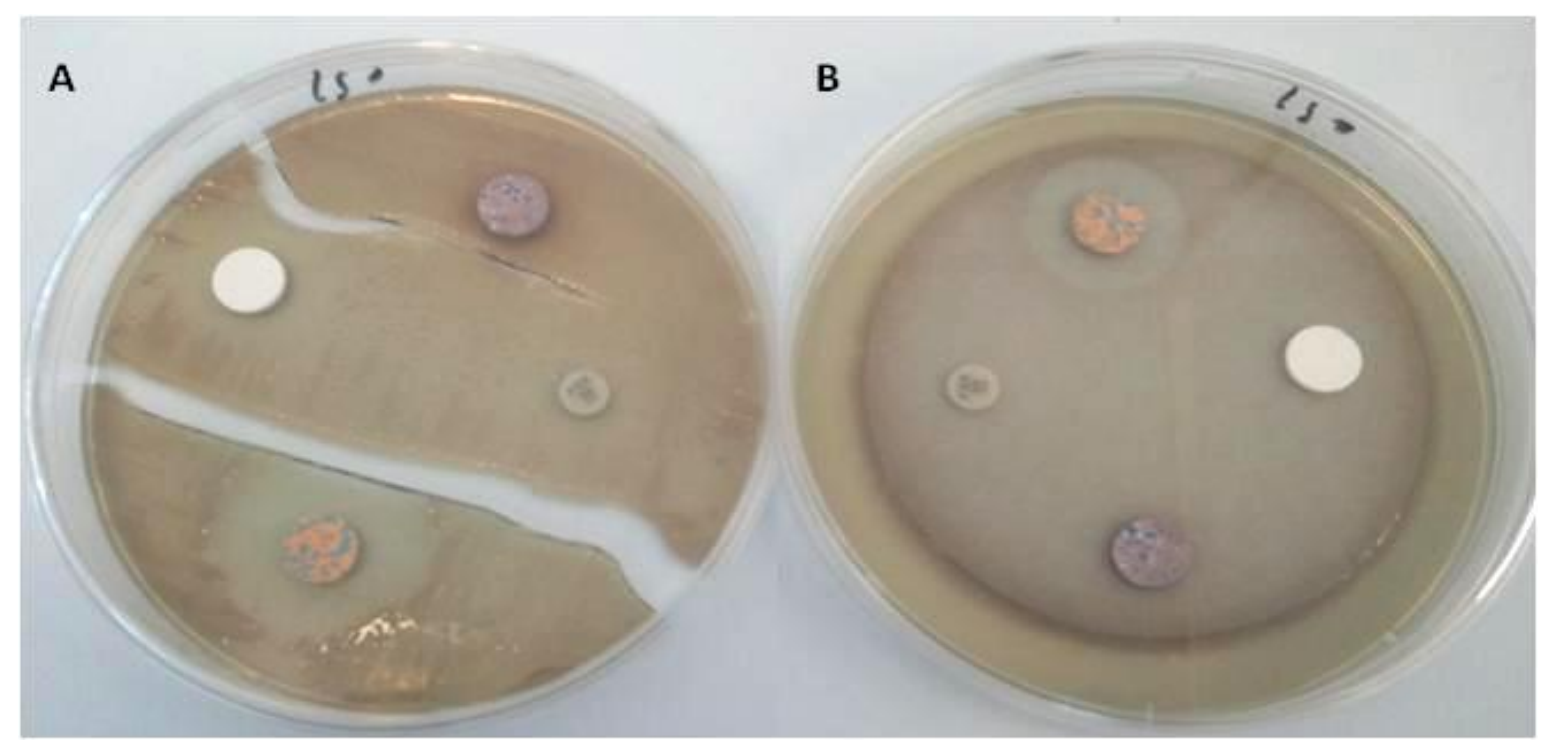

Figure 1. Comparison of the results of the antibiotic sensitivity assay for (A) planktonic culture, and (B) biofilm of Pseudomonas aeruginosa PA01. Antibiotic discs: white, chloramphenicol; orange, imipenem; small greyish, cefoxitin; and blue, vancomycin.

incubated with shaking at $120 \mathrm{rpm}$ at $37^{\circ} \mathrm{C}$. After $4 \mathrm{~h}$, the bacterial cultures were diluted to an optical density (OD) of 0.05 at $600 \mathrm{~nm}$.

\section{Initial antibiotic sensitivity tests}

For the AST, $1 \mathrm{ml}$ of the culture was added to $10 \mathrm{ml}$ of lukewarm broth, and poured over brain heart infusion (BHI)agar plates for $S$. aureus $\mathrm{SH} 1000$ and Iso-Sensitest ${ }^{\mathrm{TM}}$ Agar plates for $P$. aeruginosa PA0. Four antibiotic discs, with the following concentration were arranged over the agar: $30 \mu \mathrm{g}$ chloramphenicol, $30 \mu \mathrm{g}$ cefoxitin, 30 $\mu \mathrm{g}$ vancomycin, and $10 \mu \mathrm{g}$ imipenem. The plates were incubated at $37^{\circ} \mathrm{C}$ in air for $48 \mathrm{~h}$, after which the zone of inhibition was measured.

To perform the biofilm AST (BAST), cellulose ester discs or filters with $0.22 \mu \mathrm{m}$ pore size and $7 \mathrm{~mm}$ diameter were sterilized by autoclaving for $15 \mathrm{~min}$ at $121^{\circ} \mathrm{C}$. After cooling, the discs were soaked overnight in phosphate buffered saline. These were then dipped into overnight bacterial cultures that have been diluted to an OD 0.05 at $600 \mathrm{~nm}$. The filters were placed carefully on the surface of $\mathrm{BHI}$ agar for $S$. aureus and Iso-Sensitest agar for $P$. aeruginosa, followed by incubation at $37^{\circ} \mathrm{C}$ for $48 \mathrm{~h}$. Discs were then carefully removed and dipped in $4 \%$ human plasma in order to promote the adherence of bacteria to the membranes. After dipping, the membranes were placed onto new plates with $\mathrm{BHI}$ and IsoSensitest media. The same antibiotic discs used in the AST were then superimposed over the membrane filters and the zone of inhibition of bacterial growth was measured after $48 \mathrm{~h}$.

\section{Verification tests with strain-specific antibiotics}

Using the same protocol as mentioned earlier, a second antibiotic sensitivity test was conducted; this time the antibiotics used were specific for the bacterial strains. The following antibiotics were tested on $S$. aureus: erythromycin $(15 \mu \mathrm{g})$, ampicillin $(10 \mu \mathrm{g})$, vancomycin $(30 \mu \mathrm{g})$, gentamicin $(10 \mu \mathrm{g})$ and cefotaxime $(30 \mu \mathrm{g})$. For $P$. aeruginosa, the following antibiotic discs and concentrations were utilized: amikacin $(30 \mu \mathrm{g})$, ceftazidime $(30 \mu \mathrm{g})$, ciprofloxacin $(5$ $\mu \mathrm{g})$, imipenem $(10 \mu \mathrm{g})$, and chloramphenicol $(30 \mu \mathrm{g})$.

\section{RESULTS AND DISCUSSION}

The preliminary tests show the zones of inhibition of bacterial growth produced by the antibiotics (Figures 1 and 2). It is clear that the planktonic cultures were all sensitive to the 4 test antibiotics as compared to the biofilms. Figure 1 shows that it is only imipenem that was able to inhibit growth of the biofilm. S. aureus planktonic cultures exhibited overlapping zones while the growth zones in the biofilm were distinctly separated.

Table 1 summarizes the inhibitory action of the different antibiotics on the bacterial cultures of the two strains tested. In the preliminary trial, planktonic cultures of $S$. aureus were most sensitive to the four test antibiotics. $S$. aureus biofilm was sensitive to the action of three of the four antibiotics tested. However, the zones of inhibition were smaller than those observed for the planktonic cultures, which implies that the biofilms were able to grow despite the antibiotics. Disc zones were also observed around the antibiotics imipenem and chloramphenicol in planktonic cultures of $P$. aeruginosa. Imipenem was the only antibiotic that produced a zone of inhibition on the $P$. aeruginosa biofilm.

After the initial tests, strain-specific antibiotics were used. Planktonic $S$. aureus cultures differed in their response to the five test antibiotics. Its growth was highly inhibited by cefotaxime and ampicillin, while least inhibited by vancomycin. Compared to planktonic cultures, the biofilms were generally less affected by the antibiotics. Cefotaxime, which was highly inhibitory to planktonic growth, did not produce growth inhibition against the biofilm. The same was observed for vancomycin-treated biofilm. Erythromycin, ampicillin, and gentamicin all inhibited biofilm growth, but not as effectively as compared to 


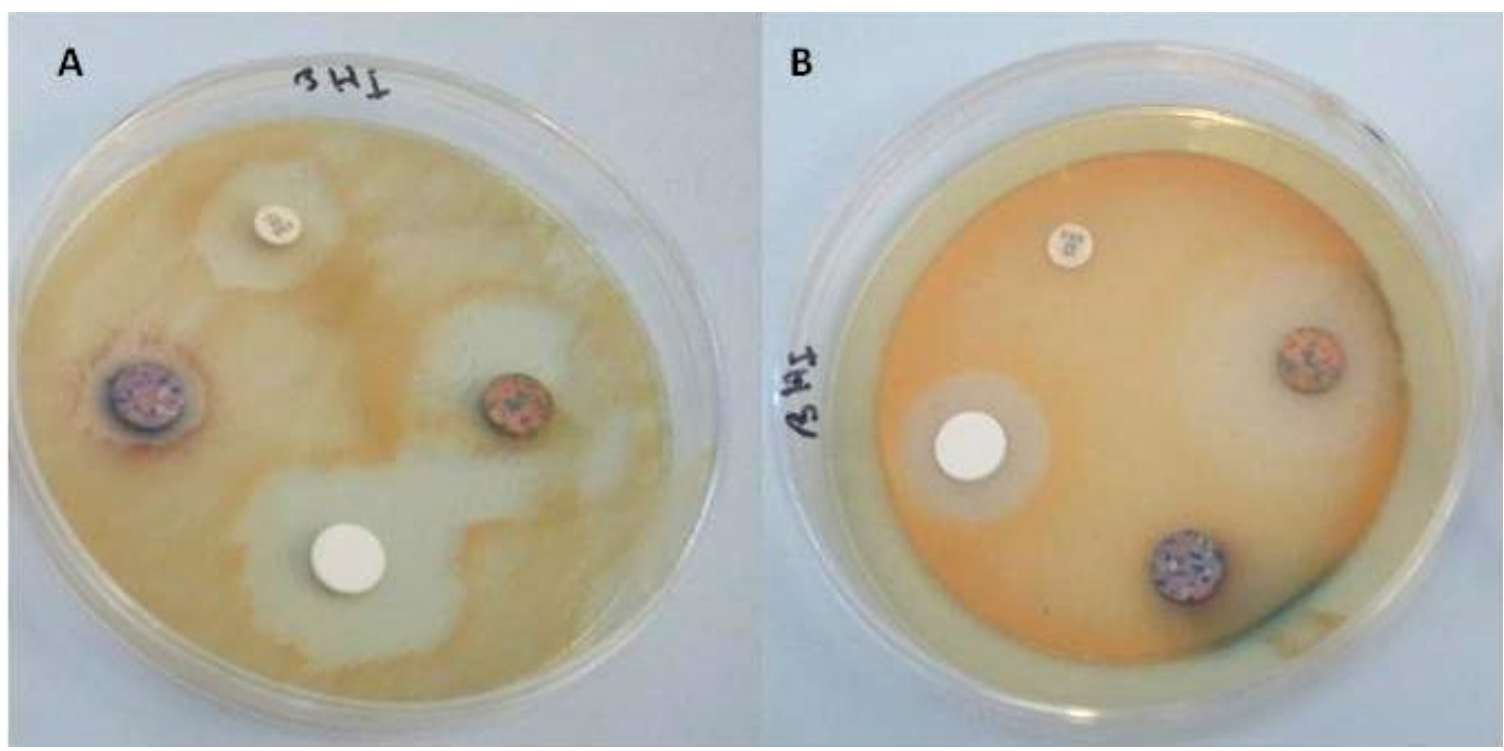

Figure 2. Comparison of the results of the antibiotic sensitivity assay for $(A)$ planktonic culture, and (B) biofilm of Staphylococcus aureus SH1000. Antibiotic discs: white, chloramphenicol; orange, imipenem; small greyish, cefoxitin; and blue, vancomycin.

Table 1. The zone of inhibition of specific antibiotics on the growth of planktonic and biofilm cultures of $S$. aureus and $P$. aeruginosa.

\begin{tabular}{llcccc}
\hline \multirow{3}{*}{ Antibiotic } & & \multicolumn{3}{c}{ Zone of inhibition (mm \pm SD) } \\
\cline { 3 - 6 } & & \multicolumn{2}{c}{ S. aureus SH1000 } & \multicolumn{1}{c}{$\boldsymbol{P}$. aeruginosa PA01 } \\
\cline { 3 - 5 } Initial test & Planktonic & Biofilm & Planktonic & Biofilm \\
\hline & Cefoxitin & $15 \pm 0.5$ & 0 & 0 & 0 \\
& Imipenem & $25 \pm 1.0$ & $20 \pm 2.5$ & $13 \pm 1.5$ & $9 \pm 0.6$ \\
& Vancomycin & $10 \pm 2.0$ & $5 \pm 1.2$ & 0 & 0 \\
& Chloramphenicol & $25 \pm 1.0$ & $15 \pm 1.2$ & $12 \pm 1.15$ & 0 \\
& & & & & \\
& Erythromycin & $15 \pm 1.0$ & $8 \pm 1.0$ & Not tested & Not tested \\
& Ampicillin & $25 \pm 1.5$ & $8 \pm 2.0$ & Not tested & Not tested \\
& Vancomycin & $8 \pm 0.6$ & $0 \pm 0$ & Not tested & Not tested \\
& Gentamicin & $15 \pm 1.5$ & $10 \pm 2.0$ & Not tested & Not tested \\
& Cefotaxime & $23 \pm 1.0$ & $0 \pm 0$ & Not tested & Not tested \\
& Amikacin & Not tested & Not tested & $20 \pm 1.7$ & $6 \pm 0.6$ \\
& Ceftazidime & Not tested & Not tested & $20 \pm 0.6$ & $6 \pm 1.5$ \\
& Ciprofloxacin & Not tested & Not tested & $30 \pm 2.5$ & $10 \pm 2.0$ \\
& Imipenem & Not tested & Not tested & $25 \pm 1.2$ & $0 \pm 0$ \\
& Chloramphenicol & Not tested & Not tested & $0 \pm 0$ & $0 \pm 0.6$ \\
\hline
\end{tabular}

*Values are means of 3 replicates. SD: Standard deviation.

their inhibition of planktonic cultures. Except for gentamicin, growth of biofilms was more than $50 \%$ higher in the presence of erythromycin and ampicillin; gentamicin proved to be most inhibitory of the antibiotics to the $S$. aureus biofilm.

In the planktonic culture of $P$. aeruginosa, four of the five test antibiotics were able to produce zones of growth inhibition ranging from 20 to $30 \mathrm{~mm}$ (Table 1). Based on the zone of inhibitions produced, the growth of biofilms was $70 \%$ more than planktonic growth despite the addition of amikacin, ceftazidime, and ciprofloxacin. In the case of imipenem, it was highly effective against planktonic cells, but not against the biofilm. This last result was not in agreement with results of the initial tests 
where imipenem inhibited biofilm growth of $P$. aeruginosa. Chloramphenicol was ineffective against $P$. aeruginosa because there was no zone of inhibition produced in both planktonic cells and biofilms.

The presence of some conflicting results indicates that more precision is necessary in carrying out the antibiotic tests. As in the case of the antibiotic sensitivity test for planktonic cultures, strict quality standards have to be met before BAST is performed. Extreme care is necessary during the preparation of the materials (that is, culture media, $\mathrm{pH}$, and antibiotic discs) for the test. In addition, the preparation of biofilms on membranes has to be perfected to ensure that growth is homogeneous and there are no contaminating strains. Although such a condition is ideal; since natural biofilms are heterogeneous (Boles et al., 2004), a single strain biofilm could prove sufficient for antibiotic sensitivity testing. The interpretation of the antibiotic disc inhibition zones has to follow standards in order to attain reliable results and to give proper recommendation. Therefore, the standards for the performance and the interpretation of BAST results also have to be developed.

\section{Conclusion}

The proposed BAST method appears to be effective in identifying antibiotics for treating biofilm infection. These preliminary results show that there is a potential of developing BAST as a sensitive assay for biofilms. More studies should be conducted for producing biofilms in vitro, standardizing methods, reagents, and conditions for BAST.

\section{REFERENCES}

Amorena B, Gracia E, Monzon M, Leiva J, Oteiza Z, Perez M, Alabart J, Hernandez-Yago J (1999). Antibiotic susceptibility assay for Staphylococcus aureus in biofilms developed in vitro. J. Antimicrob. Chemother. 44:43-55.

Bauer A, Kirby W, Sherris J, Turk M (1966). Antibiotic susceptibility testing by a standardized single disk method. Am. J. Clin. Path. 45:493-496.

Boles R, Singh P (2008). Endogenous oxidative stress produces diversity and adaptability in biofilm communities. Proc. Nat. Acad. Sci. 105(34):12503-12508.

Cerca N, Martins S, Cerca F, Jefferson KK, Pier GB, Oliveira R, Azeredo J (2005). Comparative assessment of antibiotic susceptibility of coagulase-negative Staphylococci in biofilm versus planktonic culture as assessed by bacterial enumeration or rapid XTT colorimetry. J. Antimicrob. Chemother. 56(2):331-336.

Ceri H, Olson ME, Stremick C, Read RE, Morck DW, Buret AG (1999). The Calgary Biofilm Device: New technology for rapid determination of antibiotic susceptibilities in bacterial biofilms. J. Clin. Microbiol. 37:1771-1776.

Clinical and Laboratory Standards Institute (2009). Performance standards for antimicrobial disk susceptibility tests. Approved standard M2-A10. Wayne, PA.

Jorgensen J, Ferraro M (2009). Antimicrobial susceptibility testing: a review of general principles and contemporary practices. Clin. Infect. Dis. 49(11):1749-1755.

Lewis K (2005). Persister cells and the riddle of biofilm survival. Biochemistry (Moscow). 70:267-274.

Olson ME, Ceri H, Morck DW, Buret AG, Read RR (2002). Biofilm bacteria: formation and comparative susceptibility to antibiotics. Can. J. Vet. Res. 66:86-92.

Walters M, Roe F, Bugnicourt A, Franklin M, Stewart P (2003). Contributions of antibiotic penetration, oxygen limitation, and low metabolic activity to tolerance of Pseudomonas aeruginosa biofilms to ciprofloxacin and tobramycin. Antimicrob. Agents Chemother. 47(1):317-323 